\title{
Research on Application of Wax Deposition Detection in the Nonmetallic Pipeline Based on Electrical Capacitance Tomography
}

\author{
Nan Li, ${ }^{1,2}$ Kui Liu, ${ }^{2}$ Xiangdong Yang, ${ }^{3}$ and Mingchen $\mathrm{Cao}^{2}$ \\ ${ }^{1}$ School of Automation, Northwestern Polytechnical University, Xian 710071, China \\ ${ }^{2}$ Beijing Engineering Research Center of Precision Measurement Technology and Instruments, \\ Beijing University of Technology, Beijing 100124, China \\ ${ }^{3}$ Quality Department, BAIC MOTOR, Beijing 101300, China \\ Correspondence should be addressed to Nan Li; nan.li@hotmail.co.uk
}

Received 22 April 2016; Revised 17 June 2016; Accepted 3 July 2016

Academic Editor: Lei Yuan

Copyright (C) $2016 \mathrm{Nan} \mathrm{Li}$ et al. This is an open access article distributed under the Creative Commons Attribution License, which permits unrestricted use, distribution, and reproduction in any medium, provided the original work is properly cited.

\begin{abstract}
Wax deposition detection in nonmetallic pipelines is an important requirement in the oil industry. In this paper, an ECT (electrical capacitance tomography) sensor is developed for wax deposition detection in nonmetallic pipelines. Four wax models with different concentrations were established for detection. These models were analyzed through simulations and practical experiments simultaneously and data were compared. A linear back projection algorithm is applied to reconstruct the image with both simulated and experimental data. A comparison of binary images with different concentration of stratified flow was demonstrated; this illustrates that the difference in concentration between the experimental results and profile distribution is less than $1.2 \%$. The experimental results indicate that the ECT system is valid and feasible for detecting the degree of wax deposition in the nonmetallic pipelines.
\end{abstract}

\section{Introduction}

Heat and pressure are widely used in oil and gas pipeline transmission to reduce the viscosity of crude oil and the deposition of wax. However, due to difference in radial temperature, radiation loss occurs rapidly. The wax is gradually separated out and deposited on the pipe wall, which can lead to condensate tube accidents [1]. The situation is more serious in China, as the crude oil produced in China has several distinguishing characteristics, such as high waxy distillate and low temperature viscosity. Therefore, it is important and necessary to develop a technique that can detect the deposition degree of wax inside the pipe. With consideration to the operating environment of the pipeline, as well as the cost of detection, for practical application, nondestructivebased techniques are highly recommended. Many nondestructive techniques have achieved some degree of success in the pipeline detection field, for instance, the eddy current testing method $[2,3]$, ultrasonic-based technique [4-10], radiological image method [11], and magnetic flux leakage detection method [12-14]. However, most of these techniques are applied to detect pipeline defects, and it is difficult to detect the sediment (i.e., paraffin wax) of the pipeline by using the above technologies, due to various reasons.

The detection of wax deposition in the pipeline is essentially a multiphase detection, which is composed of the wax layer, oil layer, and gas. ECT (electrical capacitance tomography) is a visual procession from measurement capacitance to object distribution. The method is a noninvasive and nondestructive technique. An ECT sensor is a typical "soft" field sensor, which means that the Electromagnetic (EM) field propagates across the entire probed volume, as shown in Figure 1. The measurement at the volume surface depends on the values of the measured quantity everywhere in the volume.

The related research began in the 1980s at the University of Manchester and, from hardware, image construction algorithms to sensor design has made considerable progress 


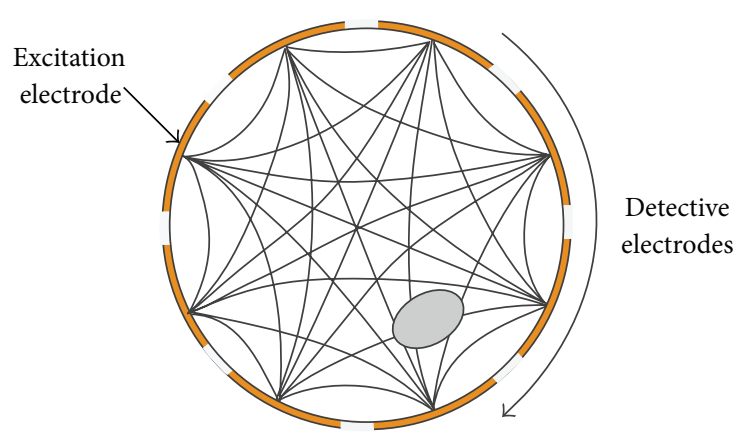

FIGURE 1: Schematic diagram of the "soft" field.

[15-21]. Johansen et al. developed a dual sensor system consisting of an 8 -electrode ECT sensor and $\gamma$-ray sensor. The new system was applied to reconstruct the image of oilgas-water three-phase flow [22]. Dyakowski et al., based on this sensor structure, presented an image reconstruction algorithm, which can be used to evaluate the concentration of a measured cross-section [23]. Schmitz and Mewes applied the ECT to the chemical industry, and the system was used to detect the transient multiphase flow in bubble columns [24]. In 2010, Yang introduced an application that used an ECT sensor to analyze the drying, pellets, and coating process of pharmaceutical fluidized beds [25]. Ismail et al. applied the ECT to the oil and gas separation process in the oil industry to determine the concentration and flow rate of each stage [26]. Al Hosani et al. suggested to use a narrowband pass filter to enhance the resolution of the produced images; the experimental results indicated that the method can improve the resolution of reconstruction images [27].

Sensor optimization is an important part of the ECT system. Xie et al. presented a uniformity of sensitivity distribution as an evaluation criterion to determine the performance of the ECT sensor [28]. Peng et al. analyzed the influence of electrode length on sensitivity distribution of the ECT sensor. The results showed that the electrode length of the ECT sensor should be at least equal to the width of the sensor electrode [29]. In 2012, Peng et al. discussed the influence of the number of the ECT sensor electrodes on the quality of image reconstruction and recommended that the number of electrodes be 12 [30]. Ren et al. presented a new algorithm to reconstruct the three-dimensional inclusion shapes; the method is valid and effective [31].

The purpose of the present paper is to develop an ECT system for wax deposition detection of a nonmetallic pipeline. The optimal sensor structure is determined based on our previous research [32]. An ECT system is demonstrated in the experimental part, and the feasibility of the system is verified based on a comparison of the reconstructed images according to simulation and experimental results.

\section{Methodology}

2.1. Wax Models. In the long-range transportation process, the temperature of the crude oil decreases and wax deposition occurs due to the thermal radiation. The permittivity of wax is 1.9 2.5. The wax is deposited on the inner wall of the pipeline, and the structure could be simplified as a stratified flow pattern in a laboratory environment. A series of wax deposition models is built and the cross-sections are shown in Figure 2. Note that the last model of Figure 2 is a special model, namely, heteromorphous flow. In order to further clarify the different structures of the wax, $u$ is defined as concentration. Note that (1) is valid for 2D problems:

$$
u=\frac{A_{\mathrm{wax}}}{A_{0}} \times 100 \%
$$

where $A_{\text {wax }}$ is the area of wax and $A_{0}$ refers to the area of sensing area, that is, the cross-section of the pipeline.

2.2. Setup of Measurement System. The ECT sensor electrodes consist of copper, and earthed shielding plates are placed around the sensor electrodes. The measurement system and the sensor structure are shown in Figure 3.

The shielding wire and BNC joints are used to prevent interference. In this study, the pipe consists of PVC material. The end guarding plate consists of copper, and the earthed shielding plate consists of aluminum. According to our previous research [32], the parameters of the ECT sensor are determined as listed in Table $1: N$ is the number of electrodes; $W$ refers to the central angle of the electrodes; and $r_{1}, r_{2}$, and $r_{3}$ are the radii of the pipe's interior and exterior and the radius of the earthed shielding plate, respectively. $L_{\text {plate }}$ represents the length of the sensor electrodes. The width of the end guarding plate is $L_{\text {end }}$, and the distance between the end guarding plate and sensor electrode is defined as $L_{\text {gap }}$.

\section{Results and Discussion}

The practical stratified models are shown in Figure 4, and the concentrations $u$ of the deposited wax are $11 \%, 18.5 \%$, $52.2 \%$, and $62.5 \%$, respectively. In order to calibrate the measurement results, two more situations should be considered, namely, those for an empty pipe and a pipe full of wax. Moreover, simulation and practical experiments are processed and analyzed simultaneously, presented, respectively, as $C_{\text {sim }}$ and $C_{m}$. The results are listed in Table 2 . The simulation model is 3D model; FEM (finite element method) is used to analyze the 


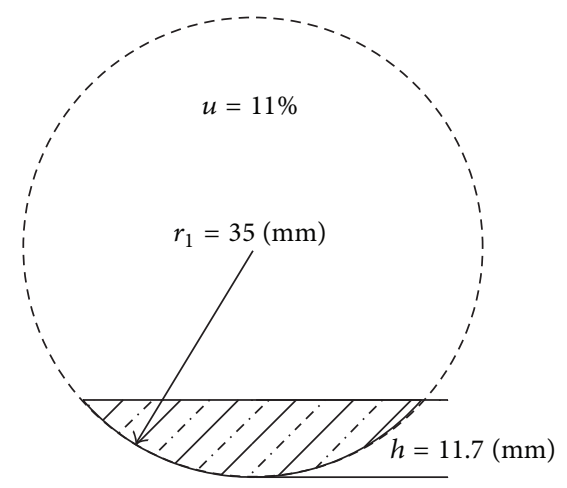

(a)

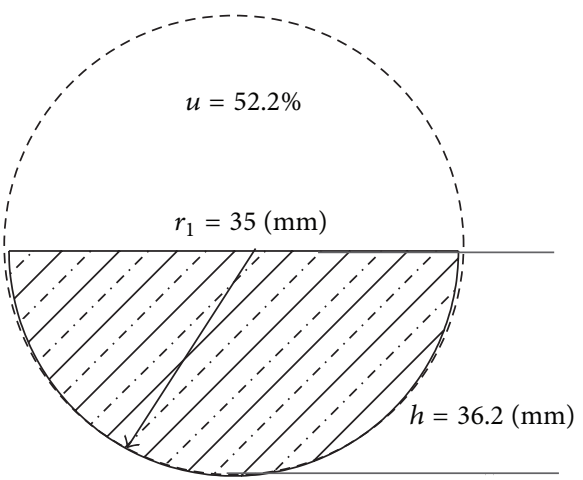

(c)

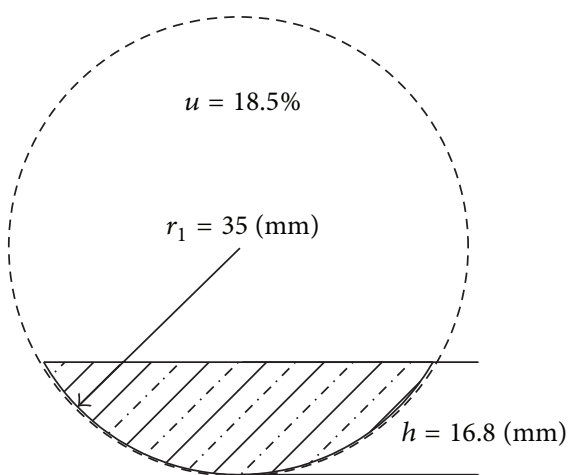

(b)

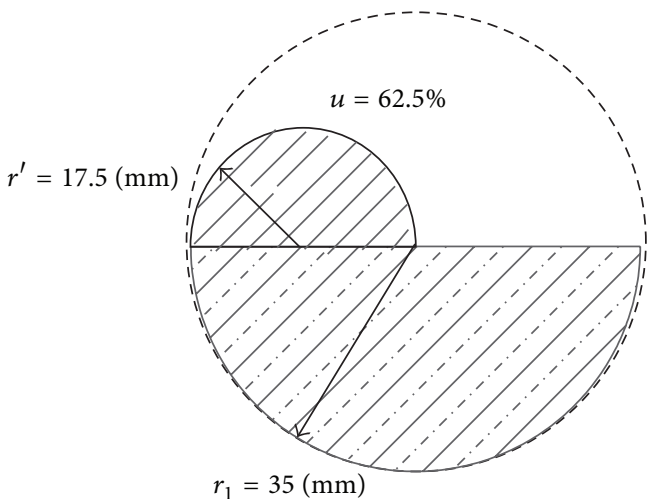

(d)

Figure 2: Different thicknesses of wax deposition models. (a) Concentration is $11 \%$. (b) Concentration is $18.5 \%$. (c) Concentration is $52.2 \%$. (d) Concentration is $62.5 \%$ (note: the last one is special stratified flow-heteromorphous flow).

TABLE 1: Parameters of the sensor.

\begin{tabular}{|c|c|c|c|c|c|c|c|c|}
\hline & \multicolumn{3}{|c|}{ Sensor electrodes } & \multicolumn{2}{|c|}{ Pipeline } & Earthed shielding plate & \multicolumn{2}{|c|}{ End guarding plate } \\
\hline Materials & \multicolumn{3}{|c|}{ Copper } & \multicolumn{2}{|c|}{ PVC } & Aluminum & \multicolumn{2}{|c|}{ Copper } \\
\hline \multirow{2}{*}{ Parameters } & $N$ & $W$ & $L_{\text {plate }}$ & $r_{1}$ & $r_{2}$ & $r_{3}$ & $L_{\text {end }}$ & $L_{\text {gap }}$ \\
\hline & 6 & $15^{\circ}$ & $105 \mathrm{~mm}$ & $35 \mathrm{~mm}$ & $40 \mathrm{~mm}$ & $50 \mathrm{~mm}$ & $20 \mathrm{~mm}$ & $10 \mathrm{~mm}$ \\
\hline
\end{tabular}

sensitivity distribution. The conditions of simulation models are the same as practical models. However, because the electrostatic module is applied, the frequency of excitation is not considered during simulation.

In order to illustrate the comparison results more intuitively, the normalized capacitances are calculated according to (2), and the results are shown in Figure 5. Consider

$$
\operatorname{nor} C=\frac{\left(C_{m}-C_{l}\right)}{\left(C_{h}-C_{l}\right)} \text {, }
$$

where nor $C$ is the normalized value and $C_{l}$ and $C_{h}$ are measured capacitance filled with low dielectric constant and high permittivity materials, respectively. For example, in this study $C_{l}$ is the capacitance when the pipe is empty, and $C_{h}$ is the capacitance when the pipe is full of wax.
According to Figure 5, the following can be derived. The same tendencies for the measurement results from the simulation and experiments are observed. For regular stratified flow patterns, the difference of the two curves decreases with the increase of concentration $u$, as shown in Figures 5(a)-5(c). The reason for this comes from two aspects: (i) the sensitivity distribution of the ECT sensor which was nonuniform; the sensitivity close to the sensor electrodes was much higher than that in the center of the sensing area; (ii) the inaccurate forward modeling or the noise in measurements. Note that the difference of the two curves increases in Figure 5(d) compared with Figure 5(c), the reason for which is that a heteromorphous model affects the measurement results.

In order to examine the effects of the design factors, it was necessary to perform the image reconstructions. Large numbers of algorithms have been developed to derive 


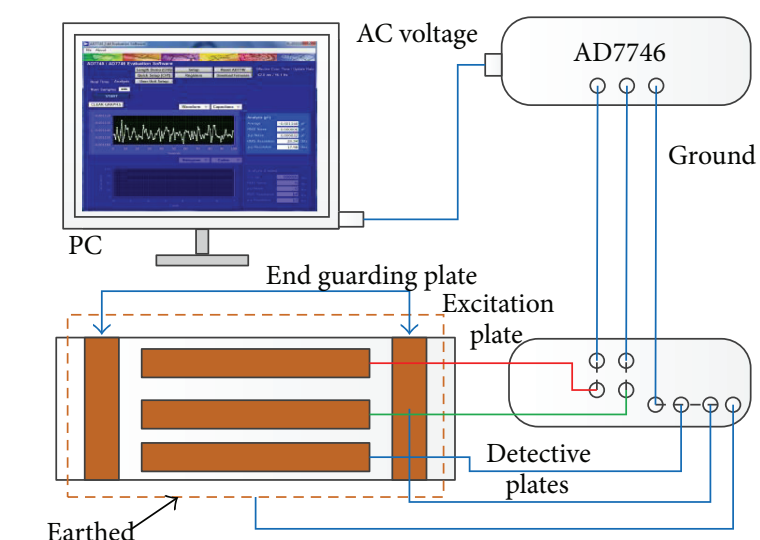
shielding plate
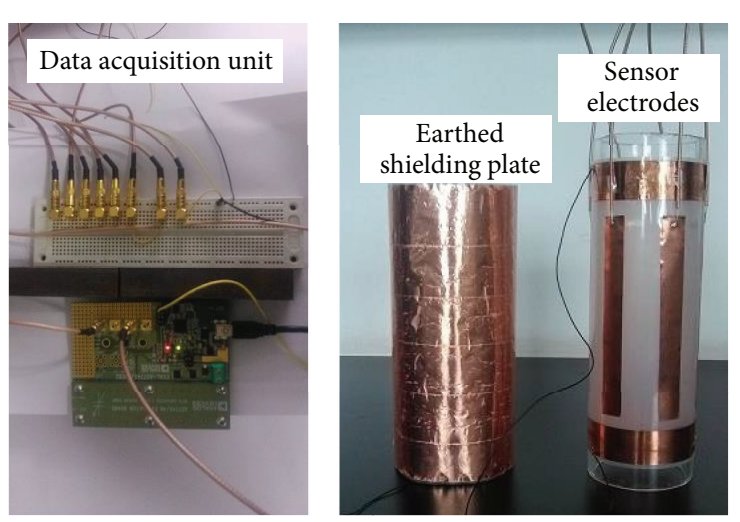

— Ground

_ Excitation

(a)

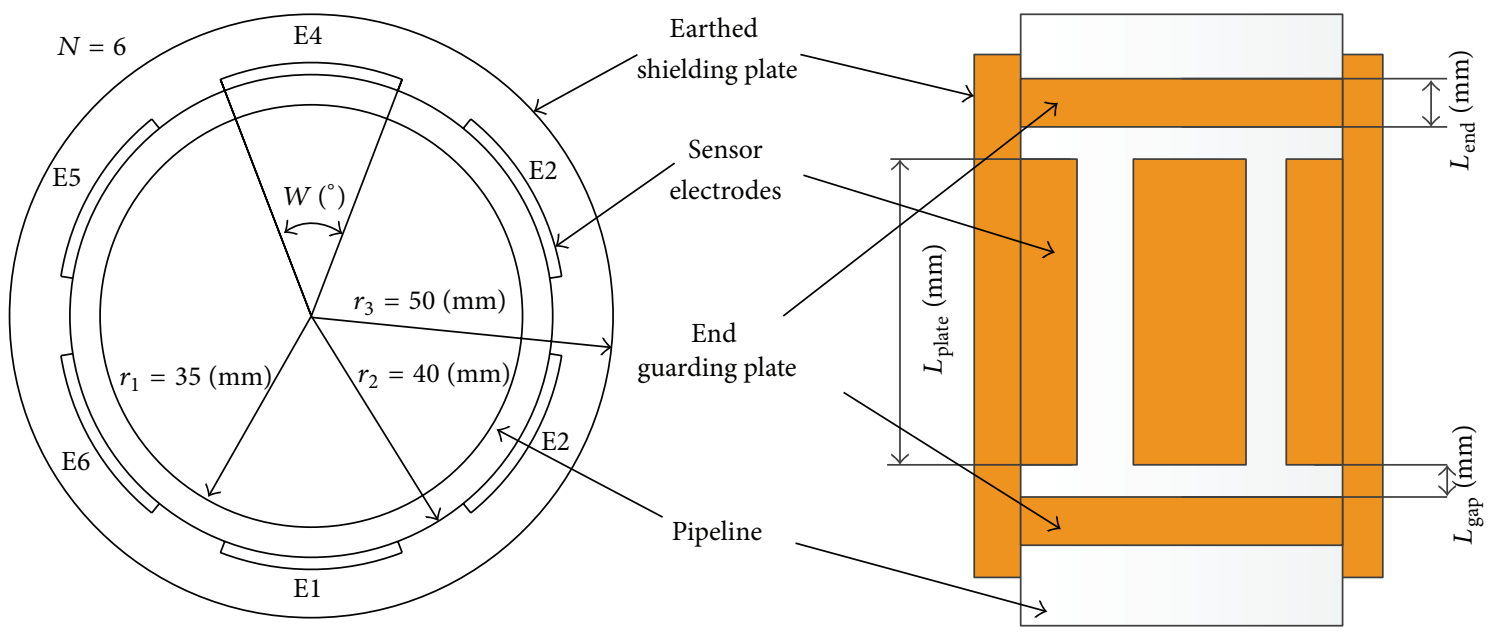

(b)

Figure 3: ECT system and sensor structure. (a) Diagram of measurement system and practical ECT sensor. (b) Parameters of the ECT sensor.

reconstructed images over the past several decades, such as linear back projection (LBP), Landweber iteration, and the sparsity-inspired image reconstruction method. In [19], Yang and Peng have analyzed the characterizations of five image reconstruction algorithms and three criteria were used to evaluate the performance of reconstructed images:

(1) relative image error,

(2) relative capacitance residual,

(3) correlation coefficient between the test object and the reconstruction.

\section{Consider}

$$
\begin{aligned}
& \text { Image error }=\left\|\frac{\hat{g}-g}{g}\right\|, \\
& \text { Capacitance residual }=\frac{\|\lambda-S \cdot \hat{g}\|}{\|\lambda\|},
\end{aligned}
$$

Correlation coefficient

$$
=\frac{\sum_{i=1}^{N}\left(\widehat{g}_{i}-\overline{\hat{g}}\right)\left(g_{i}-\bar{g}\right)}{\sqrt{\sum_{i=1}^{N}\left(\widehat{g}_{i}-\overline{\widehat{g}}\right)^{2} \sum_{i=1}^{N}\left(g_{i}-\bar{g}\right)^{2}}},
$$

where $g$ is the true permittivity distribution of the test object, $\hat{g}$ is the reconstructed permittivity distribution, and $\bar{g}$ and $\overline{\widehat{g}}$ are the mean values of $g$ and $\hat{g}$, respectively. $\lambda$ is the normalized capacitance vector, $S$ represents the normalized sensitivity matrix, and $N$ refers to the number of pixels. According to [19], the image error and correlation coefficient of LBP are better than SVD and Tikhonov and worse than Iterative Tikhonov method. The capacitance residual of LBP is similar to Iterative Tikhonov and Projected Landweber, but worse than SVD and Tikhonov methods. Due to the fact 


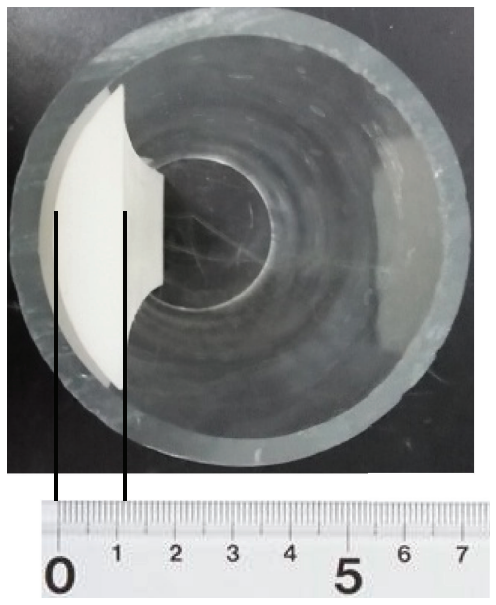

(a)

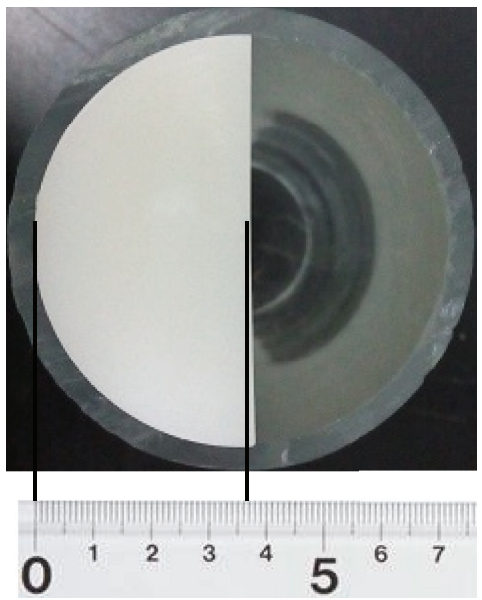

(c)

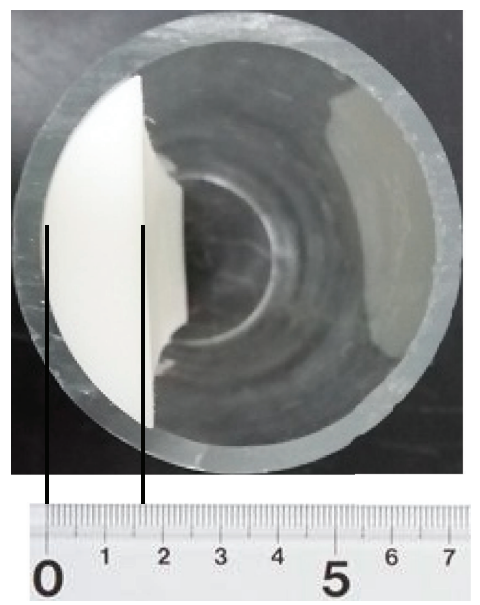

(b)

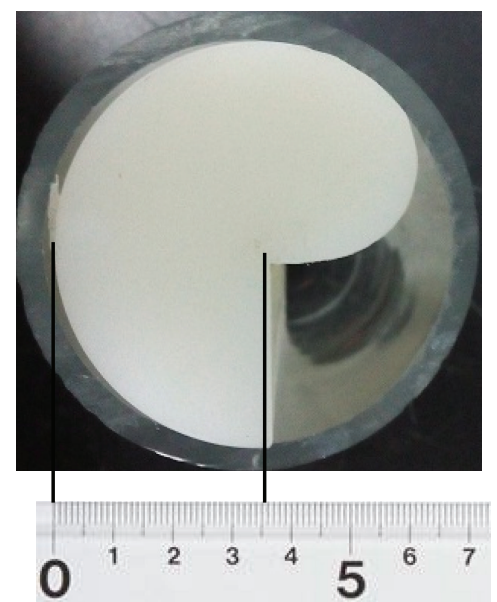

(d)

Figure 4: Practical models of stratified flow wax. (a) Concentration is $11 \%$. (b) Concentration is $18.5 \%$. (c) Concentration is $52.2 \%$. (d) Concentration is $62.5 \%$.

TABLE 2: Comparison of simulation results with experimental results.

\begin{tabular}{|c|c|c|c|c|c|c|c|c|c|c|c|c|c|}
\hline \multicolumn{2}{|c|}{$\begin{array}{c}\text { Flow } \\
\text { patterns }\end{array}$} & \multicolumn{2}{|c|}{ Empty [fF] } & \multicolumn{2}{|c|}{ Full wax [fF] } & \multicolumn{2}{|c|}{$\begin{array}{c}\text { Stratified } \\
\text { flow (a) [fF] }\end{array}$} & \multicolumn{2}{|c|}{$\begin{array}{c}\text { Stratified } \\
\text { flow (b) [fF] }\end{array}$} & \multicolumn{2}{|c|}{$\begin{array}{c}\text { Stratified } \\
\text { flow (c) }[\mathrm{fF}]\end{array}$} & \multicolumn{2}{|c|}{$\begin{array}{c}\text { Stratified } \\
\text { flow }(\mathrm{d})[\mathrm{fF}]\end{array}$} \\
\hline \multicolumn{2}{|c|}{$u$} & \multicolumn{2}{|c|}{$0 \%$} & \multicolumn{2}{|c|}{$100 \%$} & \multicolumn{2}{|c|}{$11 \%$} & \multicolumn{2}{|c|}{$18.5 \%$} & \multicolumn{2}{|c|}{$52.2 \%$} & \multicolumn{2}{|c|}{$62.5 \%$} \\
\hline & & $C_{\text {sim }}$ & $C_{m}$ & $C_{\text {sim }}$ & $C_{m}$ & $C_{\text {sim }}$ & $C_{m}$ & $C_{\text {sim }}$ & $C_{m}$ & $C_{\text {sim }}$ & $C_{m}$ & $C_{\text {sim }}$ & $C_{m}$ \\
\hline 1 & 2 & 300.9 & 288.3 & 419.8 & 382.3 & 380.0 & 335.6 & 427.6 & 350.5 & 448.9 & 383.0 & 444.8 & 385.3 \\
\hline 1 & 3 & 45.9 & 47.7 & 100.9 & 100.7 & 56.0 & 53.2 & 58.7 & 56.8 & 72.1 & 71.2 & 70.2 & 71.4 \\
\hline 1 & 4 & 30.8 & 30.5 & 70.3 & 69.3 & 37.2 & 35.8 & 39.0 & 37.1 & 42.7 & 42.7 & 45.8 & 50.4 \\
\hline 1 & 5 & 45.3 & 44.7 & 99.7 & 85.3 & 55.1 & 44.7 & 58.4 & 47.6 & 71.4 & 60.8 & 96.4 & 90.5 \\
\hline 1 & 6 & 298.0 & 264.1 & 416.5 & 351.4 & 376.0 & 317.7 & 430.4 & 335.0 & 445.3 & 358.0 & 432.0 & 346.6 \\
\hline 2 & 3 & 304.4 & 307.5 & 424.4 & 405.8 & 300.6 & 301.1 & 300.3 & 297.0 & 343.7 & 331.1 & 340.3 & 338.3 \\
\hline 2 & 4 & 45.2 & 48.7 & 99.4 & 100.8 & 43.1 & 47.6 & 44.7 & 48.1 & 56.8 & 59.2 & 57.0 & 66.1 \\
\hline 2 & 5 & 30.9 & 29.1 & 70.7 & 68.7 & 29.0 & 29.1 & 31.0 & 32.4 & 45.8 & 44.2 & 62.9 & 72.4 \\
\hline 2 & 6 & 45.1 & 46.6 & 99.3 & 99.1 & 48.3 & 60.2 & 68.0 & 77.3 & 116.0 & 110.5 & 111.9 & 104.3 \\
\hline 3 & 4 & 298.1 & 297.0 & 416.4 & 397.5 & 296.8 & 294.5 & 295.6 & 295.6 & 280.2 & 285.3 & 281.3 & 274.8 \\
\hline 3 & 5 & 45.3 & 44.3 & 99.5 & 96.6 & 43.9 & 43.2 & 42.5 & 43.5 & 38.3 & 38.4 & 47.9 & 62.4 \\
\hline 3 & 6 & 30.7 & 30.4 & 70.2 & 67.7 & 29.0 & 30.7 & 31.1 & 33.0 & 45.8 & 47.0 & 47.1 & 45.7 \\
\hline 4 & 5 & 300.4 & 295.9 & 419.6 & 390.3 & 299.6 & 296.7 & 298.7 & 284.9 & 288.1 & 285.9 & 291.7 & 334.8 \\
\hline 4 & 6 & 45.2 & 41.5 & 99.5 & 90.4 & 43.6 & 41.0 & 45.2 & 42.7 & 57.9 & 54.4 & 69.9 & 72.1 \\
\hline 5 & 6 & 298.6 & 171.5 & 417.2 & 268.7 & 296.9 & 168.4 & 298.7 & 173.2 & 340.3 & 209.1 & 397.1 & 273.4 \\
\hline
\end{tabular}




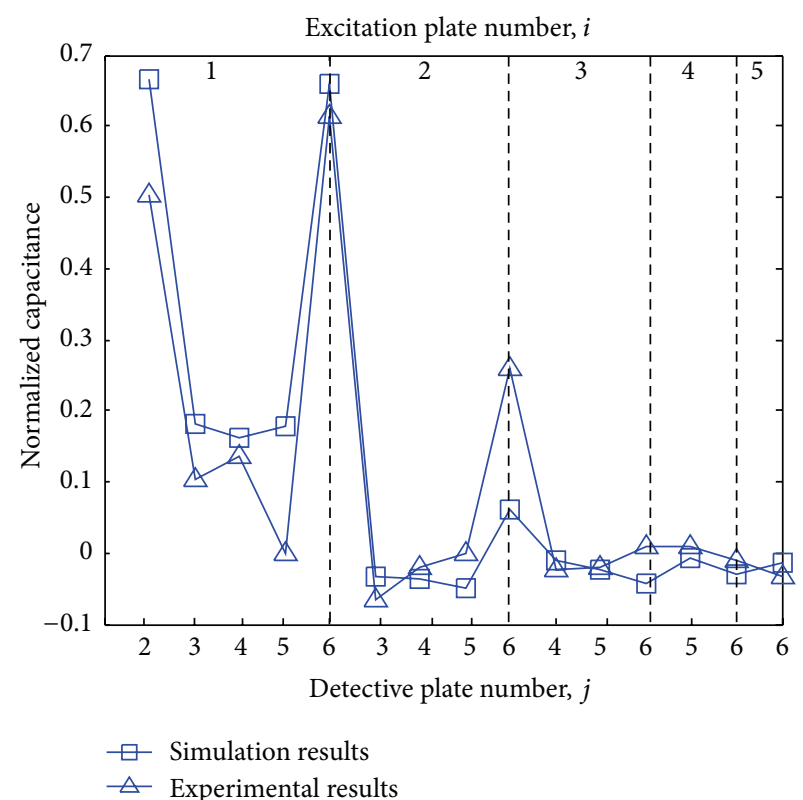

(a)

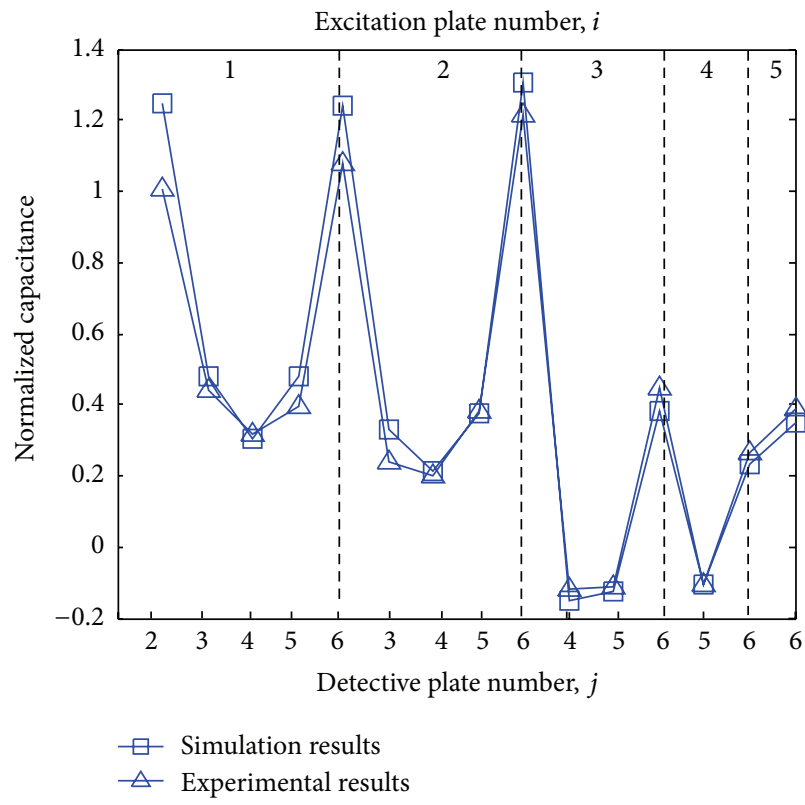

(c)

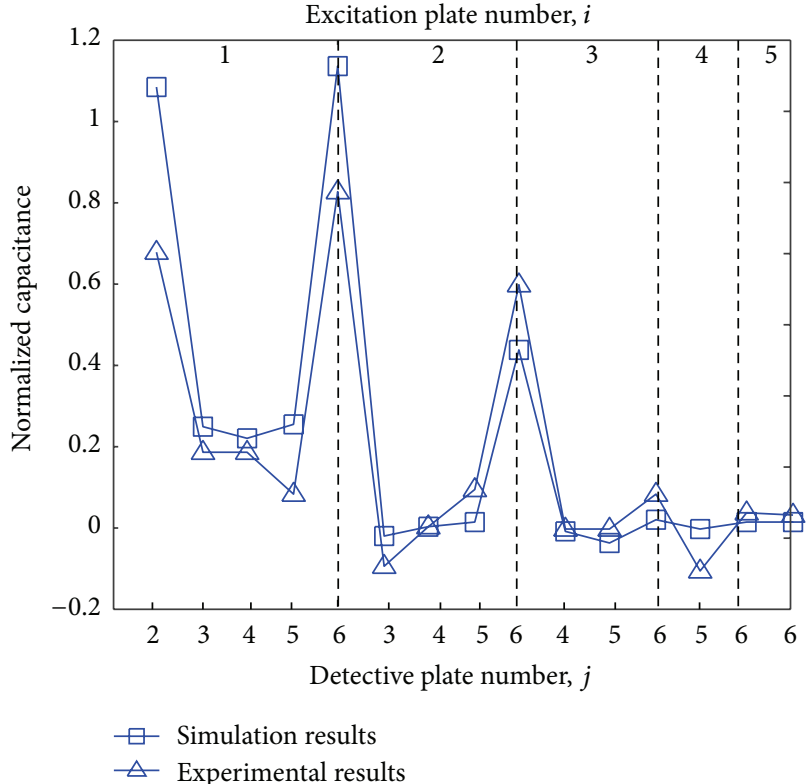

(b)

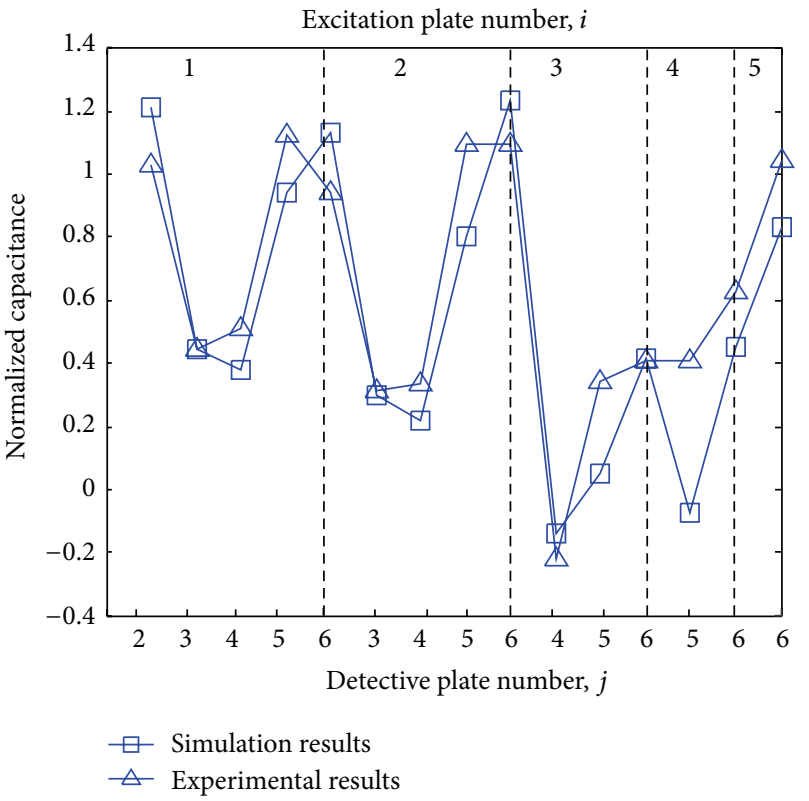

(d)

FIGURE 5: Normalized capacitance trend analysis. (a) Concentration is $11 \%$. (b) Concentration is $18.5 \%$. (c) Concentration is $52.2 \%$. (d) Concentration is $62.5 \%$.

that noniterative algorithms are typically used in practical applications and that the time consumption is less than in iterative algorithms, in order to simplify the comparison, the LBP algorithm is used to reconstruct the image [32]. Moreover, the LBP method is more suitable than iterative algorithms for dealing with stratified flow patterns. The reconstructed images are shown in Figure 6. Note that the images are based on the normalized data of the simulation and experiment data, separately. Figure 6(a) is a series of reconstructed images based on the simulation results, and
Figure 6(b) is a series of reconstructed images based on the experimental results.

The imaging results of the experiments are consistent with the reconstructed images of the simulations, which signifies that the designed ECT system is valid and feasible. For different concentrations of the wax, the reconstructed images are varied. Especially for the heteromorphous flow pattern, the shape of the semicircular convex is difficult to identify. The boundary of the heteromorphous flow pattern is difficult to determine, and the stratified flow resembles an 

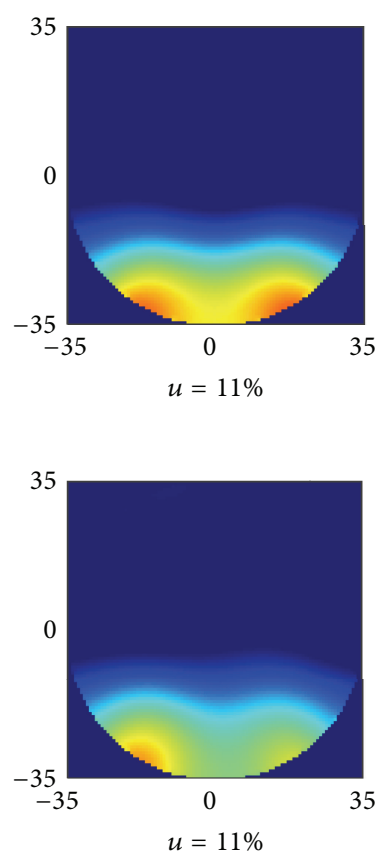
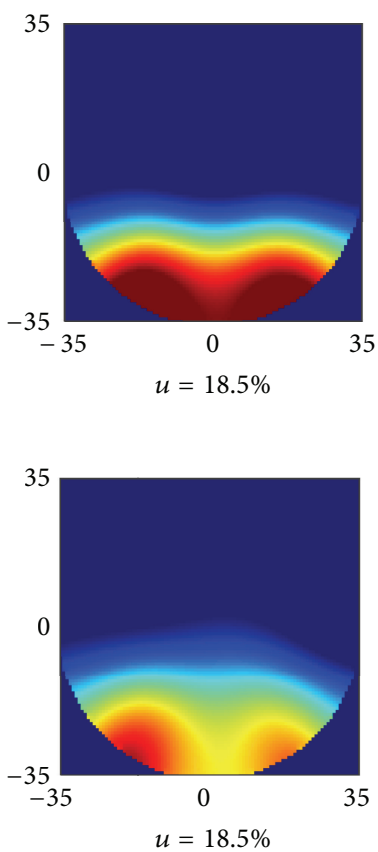

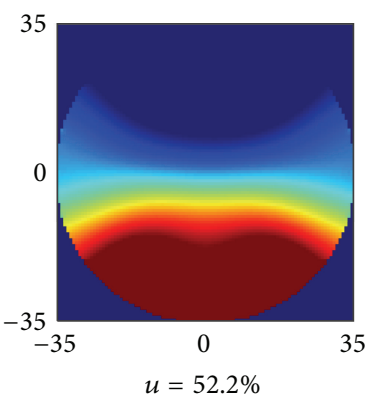

(a)

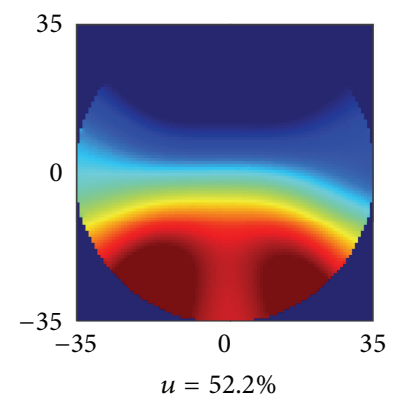

(b)
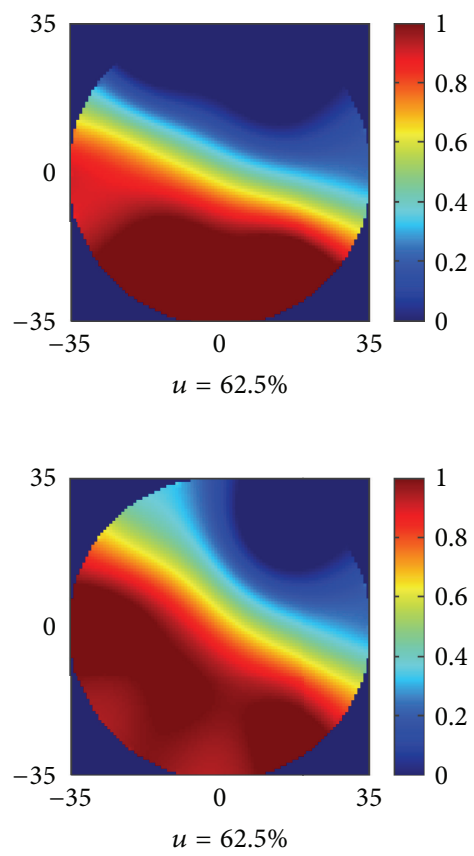

FIGURE 6: Different concentrations of stratified flow image results. (a) Simulation results. (b) Experimental results.

inclined plane. According to [19,21], this issue could be solved in terms of two aspects, namely, algorithm improvement and ECT sensor optimization. In order to evaluate the validation of using reconstructed images to identify the concentration of the wax, threshold processing was completed, and binary images of different concentrations of stratified flow are presented and compared with the standard models. The threshold processing is based on adaptive threshold filtering method which was presented by Xie et al. [33] as shown in Figure 7.

In Figure 7, $\beta_{m}$ is the initial concentration, which can be determined via a nonthreshold imaging results and experience. In this study, we define $\beta_{m}=N\left(G_{s}\left(e_{k}\right)_{\max }\right) / N\left(G_{s}\left(e_{k}\right)\right)$, where $N$ is the number of pixels, $G_{s}$ represents standard binary image, $N\left(G_{s}\left(e_{k}\right)_{\max }\right)$ refers to the number of pixels whose value is 1 in standard binary image, and $N\left(G_{s}\left(e_{k}\right)\right)$ represents the total number of pixels in standard binary image. The concentration increment $\Delta \beta=\left|\beta_{t}-\beta_{m}\right|$ and the initial value is set to 0 , where $\beta_{t}=N\left(G_{\text {new }}\left(e_{k}\right)_{\max }\right) / N\left(G_{\text {new }}\left(e_{k}\right)\right)$; $N\left(G_{\text {new }}\left(e_{k}\right)_{\text {max }}\right)$ represents number of pixels whose value is 1 in processed image and $N\left(G_{\text {new }}\left(e_{k}\right)\right)$ represents the total number of pixels in processed image. $T_{r}$ refers to threshold, and $\Delta T$ is the threshold increment whose initial value is set to 0 . Taking the flow chart as an example, each iteration step increment is 0.0005; steps can be corrected according to the actual operation. Threshold is determined by the following equation:

$$
T_{r}=G_{\min }+\Delta T\left(G_{\max }-G_{\min }\right) .
$$

TABLE 3: Contrast between standard images and reconstructed images.

\begin{tabular}{lcccc}
\hline & Model 1 & Model 2 & Model 3 & Model 4 \\
\hline $\begin{array}{l}\text { Concentration of standard } \\
\text { images }\end{array}$ & $11 \%$ & $18.5 \%$ & $52.5 \%$ & $62.5 \%$ \\
$\begin{array}{l}\text { Concentration of } \\
\text { reconstructed images }\end{array}$ & $12.2 \%$ & $19.2 \%$ & $52.97 \%$ & $62.9 \%$ \\
$\begin{array}{l}\text { Correlation coefficient } \\
\text { 9 }\end{array}$ & $93.79 \%$ & $92.78 \%$ & $98.01 \%$ & $87.04 \%$ \\
\hline
\end{tabular}

The threshold value takes the minimum pixel value as a reference; the increment is the product of pixel value difference and threshold increment. In this paper, the threshold values are $0.705,0.78,0.38$, and 0.395 , respectively, for the four reconstructed images.

The binary images of different concentrations of stratified flows are shown in Figure 8, and the concentrations and correlation coefficients are listed in Table 3. The difference of concentrations between the standard models and reconstructed images is less than $1.2 \%$, which indicates the validation of the ECT system. However, the definition of concentration is the ratio of wax deposition to the total sensing cross-section. It may be improper to evaluate the quality of reconstructed image. Therefore, the correlation coefficients of different models are listed in Table 3.

As we can see from Table 3, when concentration is $52.5 \%$ (model 3), the correlation coefficient is the best $(98.01 \%)$. 


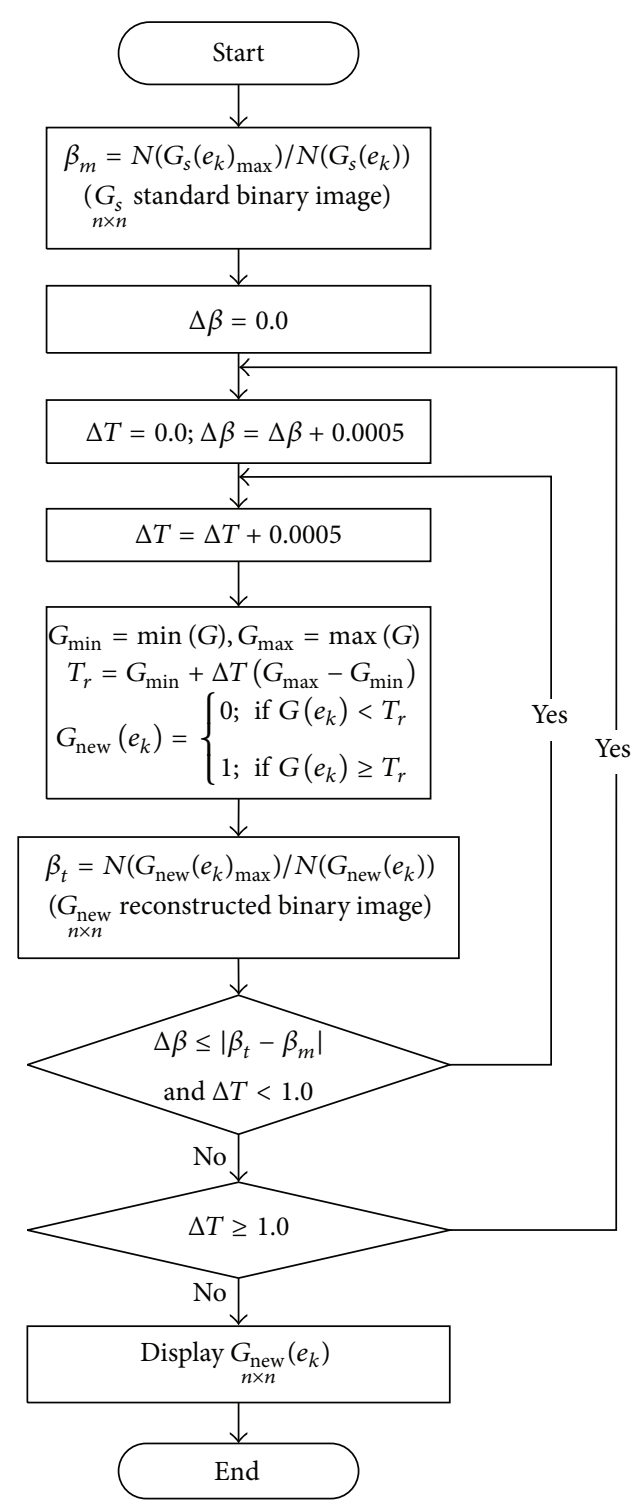

FIGURE 7: Flowchart of the linear back projection threshold processing method.

When the concentration is $11 \%$ and $18.5 \%$ (model 1 and model 2), the correlation coefficients are similar to each other and the quality of images is acceptable. However, when the concentration is $62.5 \%$ (model 4 ), the correlation coefficient is the worst $(87.04 \%)$. The main reason is the limitation of the LBP algorithm which is not good at imaging of heteromorphous flow, especially for edge imaging.

\section{Conclusions}

For wax deposition detection in nonmetallic pipelines, the use of an ECT sensor was demonstrated. LBP algorithm was applied to reconstruct images. Four models were tested, and the reconstructed images showed that the sensor is feasible. However, due to the limitations of the algorithm, it was difficult to identify the special stratified model. The boundary of the heteromorphous flow pattern was recognized as an incline in the reconstructed images. For the concentrations of $11 \%, 18.5 \%, 52.5 \%$, and $62.5 \%$, the differences between the profile and reconstructed image were $1.2 \%, 0.7 \%, 0.47 \%$, and $0.4 \%$, respectively. The correlation coefficients between standard images and reconstructed images were $93.79 \%$, $92.78 \%, 98.01 \%$, and $87.04 \%$, separately. This proves that the system designed in this paper can be applied to wax deposition detection of nonmetallic pipelines.

\section{Competing Interests}

The authors declare that there is no conflict of interests regarding the publication of this paper. 

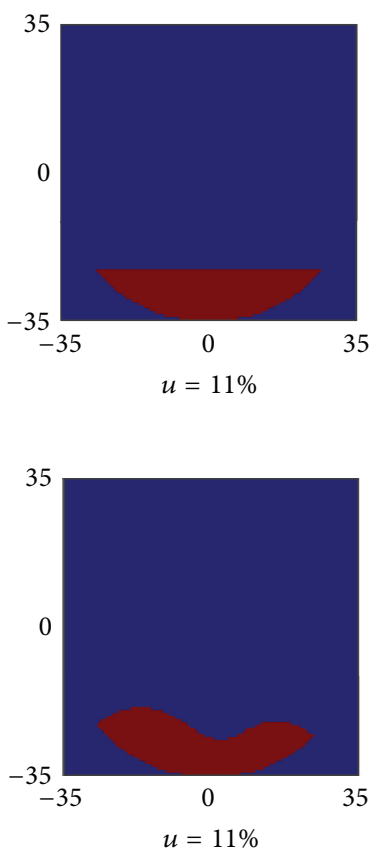
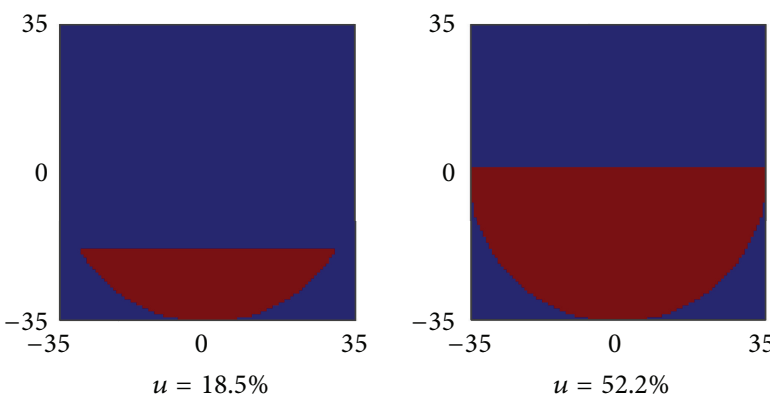

(a)

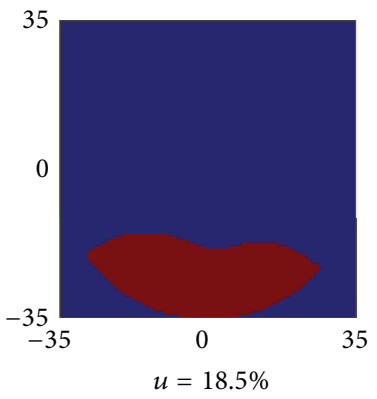

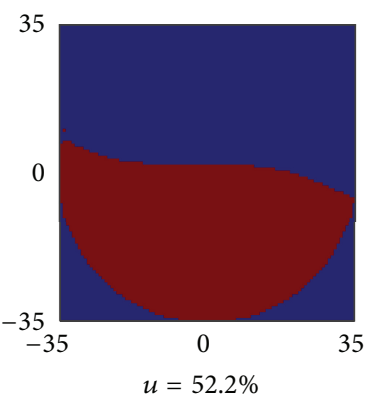
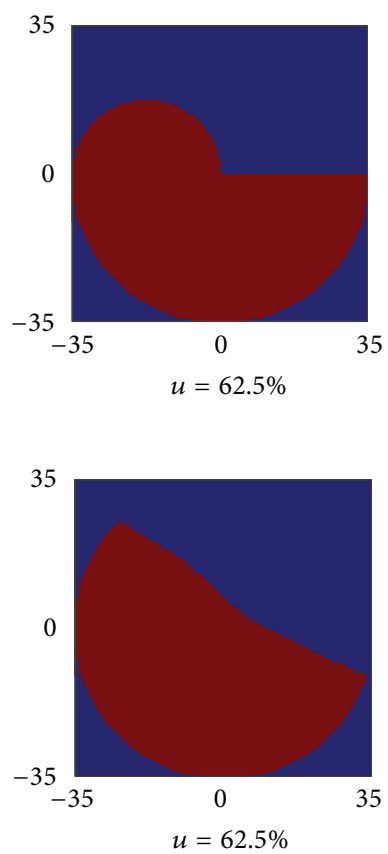

(b)

Figure 8: Binary images of different concentrations of stratified flow. (a) Profile distribution. (b) Threshold processing image based on experimental data.

\section{Acknowledgments}

This work is financially supported by the National Natural Science Foundation of China (Grants nos. 51475013, 51505013, and 51405381).

\section{References}

[1] E. D. Burger, T. K. Perkins, and J. H. Striegler, "Studies of wax deposition in the trans Alaska pipeline," Journal of Petroleum Technology, vol. 33, no. 6, pp. 1075-1086, 1981.

[2] B. Bouttier, Y. Gourbeyre, M. Hassine, J. C. Potier, and P. Bréant, "Evaluation of an intrusive technology to diagnose buried pipelines," Water Science and Technology: Water Supply, vol. 14, no. 5, pp. 931-937, 2014.

[3] C. S. Angani, D. G. Park, C. G. Kim, P. Leela, P. Kollu, and Y. M. Cheong, "The pulsed eddy current differential probe to detect a thickness variation in an insulated stainless steel," Journal of Nondestructive Evaluation, vol. 29, no. 4, pp. 248-252, 2010.

[4] G. P. P. Gunarathne and R. W. Keatch, "Novel techniques for monitoring and enhancing dissolution of mineral deposits in petroleum pipelines," Ultrasonics, vol. 34, no. 2-5, pp. 411-419, 1996.

[5] J.-W. Cheng, S.-K. Yang, and S.-M. Chiu, "The use of guided waves for detecting discontinuities in fluid-filled pipes," Materials Evaluation, vol. 65, no. 11, pp. 1129-1134, 2007.

[6] S. Shire, J. Quarini, and R. S. Ayala, "Ultrasonic detection of slurry ice flows," Proceedings of the Institution of Mechanical Engineers, Part E: Journal of Process Mechanical Engineering, vol. 219, no. 3, pp. 217-225, 2005.

[7] Z. Wu, S. Ma, Y. Chen, and Y. Wang, "Detection and identification of dents in pipelines using guided waves," Materials Evaluation, vol. 74, no. 1, pp. 79-89, 2016.
[8] G. T. Yim and T. G. Leighton, "Real-time on-line ultrasonic monitoring for bubbles in ceramic 'slip' in pottery pipelines," Ultrasonics, vol. 50, no. 1, pp. 60-67, 2010.

[9] Y. Lu, C. He, G. Song, B. Wu, C.-H. Chung, and Y.-C. Lee, "Fabrication of broadband poly(vinylidene difluoridetrifluroethylene) line-focus ultrasonic transducers for surface acoustic wave measurements of anisotropy of a (lllll 00 silicon wafer," Ultrasonics, vol. 54, no. 1, pp. 296-304, 2014.

[10] L. Yan, H. Cunfu, S. Guorong, W. Bin, C.-H. Chung, and Y.C. Lee, "Elastic properties inversion of an isotropic plate by hybrid particle swarm-based-simulated annealing optimization technique from leaky lamb wave measurements using acoustic microscopy," Journal of Nondestructive Evaluation, vol. 33, no. 4, pp. 651-662, 2014.

[11] D. Xiao, G. Zhang, Y. Liu et al., "3D detection and extraction of bladder tumors via MR virtual cystoscopy," International Journal of Computer Assisted Radiology and Surgery, vol. 11, no. 1, pp. 89-97, 2016.

[12] M. Ricci, A. Ficola, M. L. Fravolini et al., "Magnetic imaging and machine vision NDT for the on-line inspection of stainless steel strips," Measurement Science and Technology, vol. 24, no. 2, Article ID 025401, 2013.

[13] Y. Shi, C. Zhang, R. Li, M. Cai, and G. Jia, "Theory and application of magnetic flux leakage pipeline detection," Sensors, vol. 15, no. 12, pp. 31036-31055, 2015.

[14] R. Wagner, O. Goncalves, A. Demma, and M. Lowe, "Guided wave testing performance studies: comparison with ultrasonic and magnetic flux leakage pigs," Insight, vol. 55, no. 4, pp. 187196, 2013.

[15] S. M. Huang, A. B. Plaskowski, C. G. Xie, and M. S. Beck, "Tomographic imaging of two-component flow using capacitance sensors," Journal of Physics E: Scientific Instruments, vol. 22, no. 3, pp. 173-177, 1989. 
[16] C. G. Xie, A. L. Stott, S. M. Huang, A. Plaskowski, and M. S. Beck, "Mass-flow measurement of solids using electrodynamic and capacitance transducers," Journal of Physics E: Scientific Instruments, vol. 22, no. 9, pp. 712-719, 1989.

[17] H. Wang, W. Yin, W. Q. Yang, and M. S. Beck, "Optimum design of segmented capacitance sensing array for multi-phase interface measurement," Measurement Science and Technology, vol. 7, no. 1, pp. 79-86, 1996.

[18] W. Q. Yang, "Modelling of capacitance tomography sensors," IEE Proceedings-Science, Measurement and Technology, vol. 144, no. 5, pp. 203-208, 1997.

[19] W. Q. Yang and L. Peng, "Image reconstruction algorithms for electrical capacitance tomography," Measurement Science and Technology, vol. 14, no. 1, pp. R1-R13, 2003.

[20] Y. Li and W. Q. Yang, "Virtual electrical capacitance tomography sensor," Journal of Physics: Conference Series, vol. 15, no. 1, pp. 183-188, 2005.

[21] W. Q. Yang, "Design of electrical capacitance tomography sensors," Measurement Science and Technology, vol. 21, no. 4, Article ID 042001, 2010.

[22] G. A. Johansen, T. Frøystein, B. T. Hjertaker, and Ø. Olsen, "A dual sensor flow imaging tomographic system," Measurement Science and Technology, vol. 7, no. 3, pp. 297-307, 1996.

[23] T. Dyakowski, G. A. Johansen, B. T. Hjertaker, D. Sankowski, V. Mosorov, and J. Wlodarczyk, "A dual modality tomography system for imaging gas/solids flows," Particle \& Particle Systems Characterization, vol. 23, no. 3-4, pp. 260-265, 2006.

[24] D. Schmitz and D. Mewes, "Tomographic imaging of transient multiphase flow in bubble columns," Chemical Engineering Journal, vol. 77, no. 1-2, pp. 99-104, 2000.

[25] W. Yang, "Imaging pharmaceutical fluidised beds by electrical capacitance tomography," in Proceedings of the IEEE International Conference on Imaging Systems and Techniques (IST '10), pp. 52-56, Thessaloniki, Greece, July 2010.

[26] I. Ismail, J. C. Gamio, S. F. A. Bukhari, and W. Q. Yang, "Tomography for multi-phase flow measurement in the oil industry," Flow Measurement and Instrumentation, vol. 16, no. 2-3, pp. 145-155, 2005.

[27] E. Al Hosani, M. Zhang, and M. Soleimani, "A limited region electrical capacitance tomography for detection of deposits in pipelines,' IEEE Sensors Journal, vol. 15, no. 11, pp. 6089-6099, 2015.

[28] C. G. Xie, A. L. Stott, A. Plaskowski, and M. S. Beck, "Design of capacitance electrodes for concentration measurement of twophase flow," Measurement Science and Technology, vol. 1, no. 1, pp. 65-78, 1990.

[29] L. Peng, C. Mou, D. Yao, B. Zhang, and D. Xiao, "Determination of the optimal axial length of the electrode in an electrical capacitance tomography sensor," Flow Measurement and Instrumentation, vol. 16, no. 2-3, pp. 169-175, 2005.

[30] L. Peng, J. Ye, G. Lu, and W. Yang, "Evaluation of effect of number of electrodes in ECT sensors on image quality," IEEE Sensors Journal, vol. 12, no. 5, pp. 1554-1565, 2012.

[31] S. Ren, Y. Xu, C. Tan, and F. Dong, "Reconstructing the geometric configuration of three dimensional interface using electrical capacitance tomography," International Journal for Numerical Methods in Engineering, vol. 96, no. 10, pp. 628-644, 2013.

[32] N. Li, X. Yang, Y. Gong, and P. Wang, "Enhancing electrical capacitance tomographic sensor design using fuzzy theory based quantifiers," Measurement Science and Technology, vol. 25, no. 12, Article ID 125401, 2014.
[33] C. G. Xie, A. Plaskowski, and M. S. Beck, "8-electrode capacitance system for two-component flow identification. part 1 : tomographic flow imaging," IEE Proceedings A: Physical Science. Measurement and Instrumentation. Management and Education. Reviews, vol. 1, pp. 173-183, 1992. 


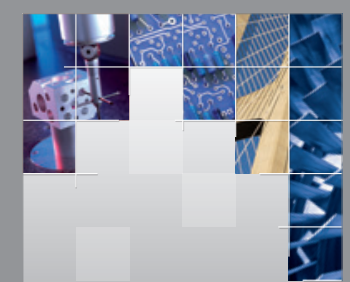

\section{Enfincering}
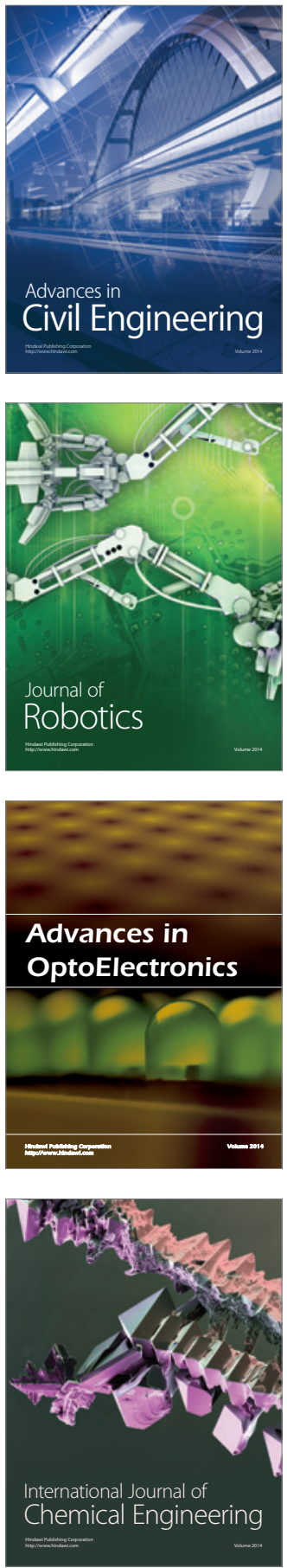

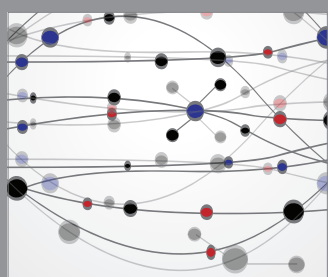

The Scientific World Journal

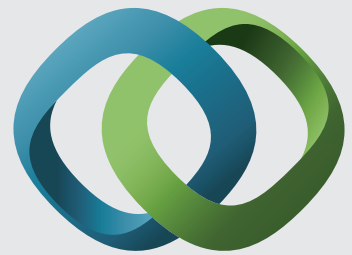

\section{Hindawi}

Submit your manuscripts at

http://www.hindawi.com
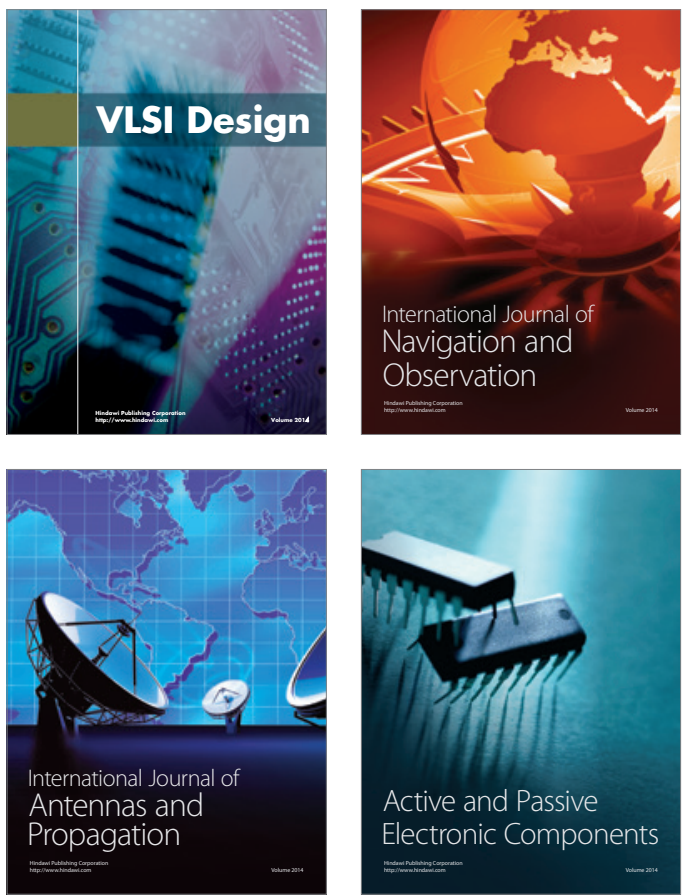
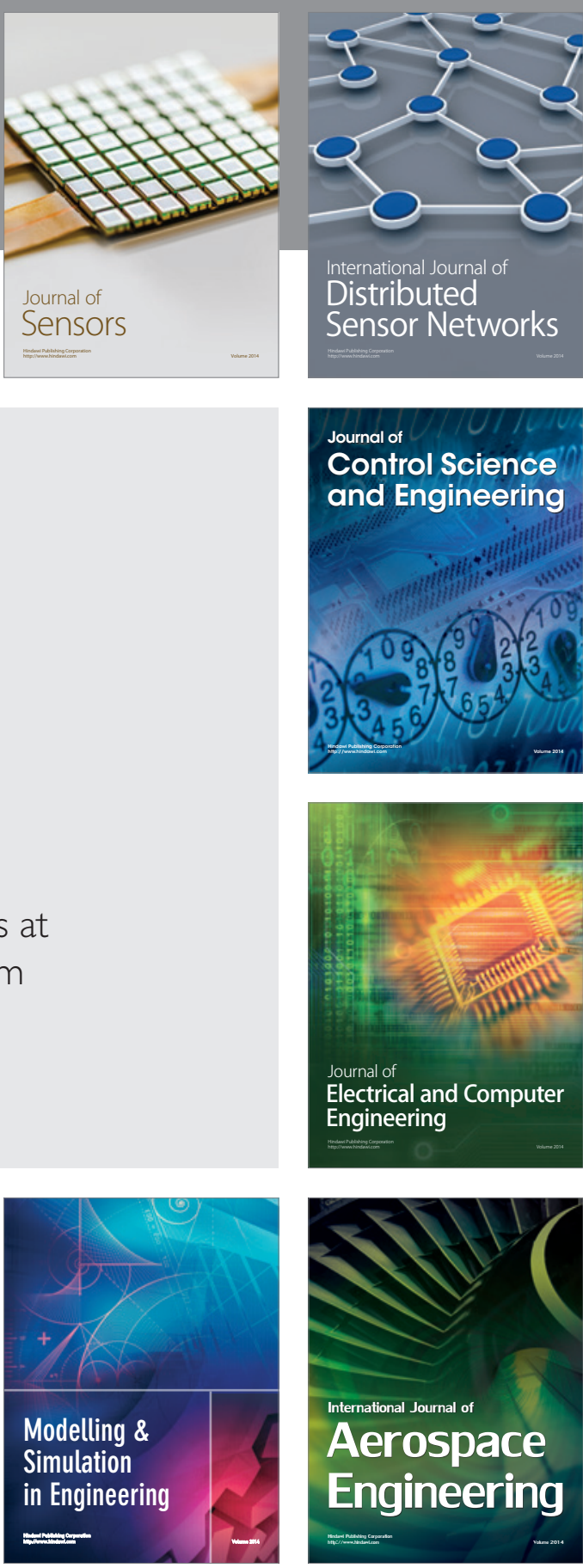

International Journal of

Distributed

Sensor Networks

Journal of

Control Science

and Engineering
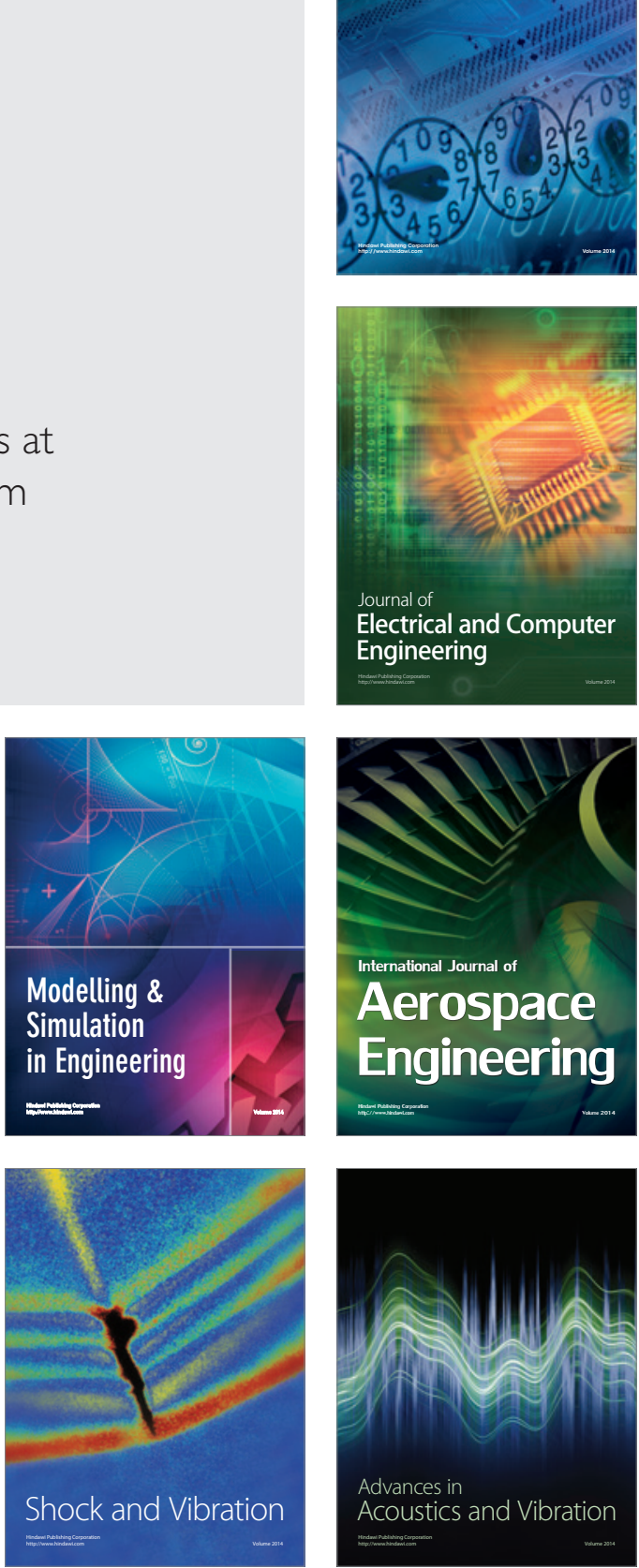